\title{
Trend analysis of palliative care consultation service for terminally ill non-cancer patients in Taiwan: a 9-year observational study
}

\author{
Lian-Shin Lin' ${ }^{1}$ Ling-Hui Huang ${ }^{1}$, Yu-Chen Chang ${ }^{2}$, Chun-Li Wang ${ }^{3,4}$, Lung-Chun Lee ${ }^{3}$, Chung-Chieh Hü ${ }^{3}$,
} Pi-Shan $\mathrm{Hsu}^{3}$ and Wei-Min $\mathrm{Chu}^{3,5,6,7,8^{*}}$

\begin{abstract}
Backgrounds: Early integration of palliative care for terminally ill non-cancer patients improves quality of life. However, there are scanty data on Palliative Care Consultation Service (PCCS) among non-cancer patients.

Methods: In this 9-year observational study Data were collected from the Hospice-Palliative Clinical Database (HPCD) of Taichung Veterans General Hospital (TCVGH). Terminally ill non-cancer patients with 9 categories of diagnoses who received PCCS during 2011 to 2019 were enrolled. Trend analysis was performed to evaluate differences in categories of diagnosis throughout study period, duration of PCCS, patient outcomes, DNR declaration, awareness of disease by patients and families before and after PCCS.

Results: In total, 536 non-cancer patients received PCCS from 2011 to 2019 with an average age of 70.7 years. The average duration of PCCS was 18.4 days. The distributions of age, gender, patient outcomes, family's awareness of disease before PCCS, and patient's awareness of disease after PCCS were significantly different among the diagnoses. Organic brain disease and Chronic kidney disease (CKD) were the most prevalent diagnoses in patients receiving PCCS in 2019. For DNR declaration, the percentage of patients signing DNR before PCCS remained high throughout the study period (92.8\% in 2019). Patient outcomes varied according to the disease diagnoses.

Conclusion: This 9-year observational study showed that the trend of PCCS among non-cancer patients had changed over the duration of the study. An increasing number of terminally ill non-cancer patients received PCCS during late life, thereby increasing the awareness of disease for both patients and families, which would tend to better prepare terminally ill patients for end-of-life as they may consider DNR consent. Early integration of PCCS into ordinary care for terminally non-cancer patients is essential for better quality of life.
\end{abstract}

Keywords: Palliative care consultation service, Non-cancer, Do-not-resuscitate, Awareness

\section{Background}

The world is rapidly aging [1]. Because of improvements in public health and medical interventions, life expectancy has increased worldwide in recent decades, and

*Correspondence: williamchu0110@gmail.com

${ }^{3}$ Department of Family Medicine, Taichung Veterans General Hospital,

Taichung, Taiwan

Full list of author information is available at the end of the article continues to rise in many countries [2]. Thus, causes of death from communicable causes to non-communicable diseases are also changing due to increased life expectancy [3]. Cancer accounts for a large proportion of deaths annually, but non-cancer terminal disease also produces disease burden and poor quality of life during late life [4].

Palliative care has been demonstrated to relieve symptoms near end-of-life [5], to overcome psychological 
distress [6], and to improve quality of life for cancer patients and their family members $[7,8]$. In recent years, the importance of palliative care for non-cancer terminally ill patients has been brought to light in multiple studies [9-11]. Studies have shown that palliative care among non-cancer patients can reduce rates of emergency department visits, admissions to hospital, and admissions to the intensive care unit during end-of-life [12]. A previous study also showed that early integration of palliative care for patients with diseases other than cancer can improve breathlessness [13], increase DoNot-Resuscitate (DNR) consent, and increase patients' and families' recognition of the diagnosis [14].

Multiple palliative care services have been initiated in recent years, including inpatient care in palliative care unit [15], palliative home care service [16] and palliative care consultation service (PCCS) [17]. A previous study has shown that PCCS during hospitalization for cancer patients can improve patients' and families' awareness of disease diagnosis and prognosis, and also increase consent to DNR [18]. Also, symptom control was better when receiving PCCS among cancer patients [19].

Palliative care service has been implemented in Taiwan's National Health Insurance (NHI) since 1996, and includes palliative home care (introduced in 1996), palliative inpatient care (2000), and PCCS (2005) [20]. In 2009, the service was expanded by allowing non-cancer terminally ill patients into the PCCS service, which is covered by the NHI program. When non-cancer terminally ill patients are admitted to hospital, they are cared for by a disease specialist as well as a palliative care specialist and a palliative care team, consisting of a nurse, a consulting psychologist, a social worker, and a volunteer. Since 2011, Taiwan has implemented significant environmental changes in palliative care, including the amendments to "Hospice Palliative Care Act" in 2011 and 2013 [21], and further announcement and execution of the "Patient Right to Autonomy Act" in 2016 and 2019 [22].

However, there are few data on PCCS for non-cancer terminally ill patients, especially in Asian countries. Thus, it's difficult to evaluate the impact of PCCS on the noncancer population. In this 9-year observational study, we aimed to evaluate the trend and impact of PCCS, including trend of diagnosis of non-cancer terminal disease, duration of PCCS in different disease categories, DNR declaration before and after PCCS, patients' and families' awareness of disease before and after PCCS, and patient outcomes among non-cancer terminally-ill patients.

\section{Methods}

Data sources

Data were collected from the Hospice-Palliative Clinical Database (HPCD) of Taichung Veterans General
Hospital (TCVGH). TCVGH is the only public tertiary medical center in central Taiwan with more than 1500 beds in the hospital. The palliative care team in TCVGH was established in 2003, with team members that include physicians, nurses, consulting psychotherapists, social workers, spiritual therapists, art therapists and trained volunteers. The palliative care team provides comprehensive hospice-palliative care in 3 different settings, including inpatient palliative care service, PCCS, and palliative home care service.

\section{Study group identification}

We enrolled terminally ill non-cancer patients who were admitted to TCVGH and referred to PCCS from Jan 2011 to Dec 2019. Since 2009, there were 9 specific diagnosis, including dementia, other brain disease (terminal condition such as severe stroke, severe brain injury, multiple sclerosis, Parkinson's disease and Huntington's disease), heart failure, chronic obstructive pulmonary disease (COPD), other diseases of the lung, chronic liver disease or cirrhosis, acute kidney injury, chronic kidney disease and motor neuron disease, which were eligible for PCCS coverage by Taiwan's NHI. For non-cancer terminally ill patients in need, his/her visiting staff would consult doctor of PCCS team initially. During consultation, the palliative care physician and nurse went to visit the patient and recorded their chief complaints, present illnesses, active problems, and previous experiences of treatment, and initiated talks on the value and preference to the patient and family. Also, after carefully evaluation, suggestion regarding patient's medication, physical care and mental care would be given by PCCS team members to patient's original care team.

\section{Research variables}

All data of enrolled patients were collected and extracted from the HPCD, including age at admission, gender, major diagnosis, date of the last admission, date of PCCS enrollment, duration of PCCS service, DNR order status, date of DNR declaration, patients' and families' awareness of disease before and after PCCS, patient outcomes, and date of discharge or death.

The data for the duration of PCCS was determined by the interval between the date of first enrollment in PCCS and date of discharge or PCCS termination. DNR order rate (in percent) was calculated as the number of patients who signed a DNR order divided by the number of patients who received PCCS each year. The number of DNR declaration before PCCS and after PCCS was also documented. The awareness of disease was assessed by PCCS nursing staff for each patient and one of their family before and after PCCS. 


\section{Statistical analysis}

Statistical analyses were performed using SAS version 9.4 (Statistical Analysis Software 9.4, SAS Institute Inc., Cary, North Carolina, USA). Gender distributions, diagnosis distributions, DNR rates, awareness of disease and patient outcomes were analyzed using chi-square tests. A two-tailed $p$ value $<0.05$ was considered statistically significant.

\section{Results}

Table 1 shows the trends of all terminally ill non-cancer patients who were admitted to TCVGH and received PCCS during hospitalization. In total, 536 patients were enrolled from 2011 to 2019 with an average age of 70.7 years. The average duration of PCCS was 18.4 days. The percentages of DNR declaration before and after PCCS was 82.8 and $15.1 \%$, respectively. The DNR order status as well as the patient outcomes differed significantly between different years.

Supplementary Fig. 1 showed trends of all terminally ill non-cancer patients who received PCCS and not received PCCS. In total, 4153 patients were died of non-cancer diseases of 9 categories. From 2011 to 2019, PCCS rate elevated from $0.36 \%$ to $27.71 \%$ and the average PCCS rate of non-cancer terminally patients was $12.9 \%$.

Table 2 shows the descriptive data by different categories of non-cancer diseases. The distributions of age, gender, patient outcomes, family's awareness of disease before PCCS, and patient's awareness of disease after PCCS were significantly different among the different diagnoses. However, the duration in days for receiving PCCS and DNR order status were not significantly different.

Patients' and families' awareness of disease are shown in Table 3. Families' awareness of disease before PCCS and patients' awareness of disease after PCCS were significantly different in different disease categories. After PCCS, families' awareness of disease were nearly $100 \%$ for all disease categories, however, patients' awareness of disease after PCCS were still low for some disease categories, especially for dementia (40\%) and COPD (43\%).

Figure 1 summarizes the trends of patients in different categories of diseases and receiving PCCS from 2011 to 2019. There was a surge of patients with organic brain disease in 2015, and the number of patients with chronic kidney disease increased gradually from 2015 to 2019 . Figure 2 shows the DNR status annually. The percentage of patients signing a DNR before PCCS remained high throughout the duration of the study ( $92.8 \%$ in 2019), but the percentage of those patients who did not sign a DNR before PCCS, but signed after PCCS was also high (100\% in 2019).
The awareness of disease among patients and families is shown in Fig. 3. Awareness of disease among family members was consistently higher than that of patients. Both patients' and families' awareness of disease significantly increased after PCCS. Figure 4 shows the trend of difference $s$ in awareness of disease between patients and families before and after PCCS. From 2011 to 2019, the difference decreased significantly especially before PCCS.

\section{Discussion}

The main findings of this 9-year observational study are as follows:

1. The number of non-cancer patients receiving PCCS increased during the past decade.

2. The trend of patients' non-cancer terminal diagnosis in PCCS program changed. Since 2015, organic brain disease was the leading diagnosis of non-cancer patients receiving PCCS. However, chronic kidney disease has increased rapidly since 2017.

3. Patient outcomes differed among the various categories of non-cancer diseases.

4. An increasing number of non-cancer patients signed a DNR consent form before PCCS, but for those who did not sign a DNR consent form, most of them signed after PCCS.

5. PCCS increased disease awareness in both patients and their families. PCCS decreased the difference in disease awareness between patients and families The difference in disease awareness between patients and families before and after PCCS gradually converged.

In our study, the number of non-cancer patients receiving PCCS increased, and rate of receiving PCCS among non-cancer terminally ill patients was also increased. More that, we also found increased service of palliative home care and palliative inpatient care during study period (Supplementary Fig. 2). Among the 3 services of palliative care, the increase of PCCS was the most significant, compare to palliative home care and palliative inpatient care, suggesting that PCCS is a good portal for non-cancer terminally ill patients who have needs for palliative care. This was consistent with a previous study which showed that non-cancer terminal patients' need for palliative care was increasing. Gadoud et al. observed that palliative care increased among patients with chronic obstructive lung disease and heart failure in the UK from 2009 to 2014 [9]. Hess et al. in Germany found that the proportion of non-cancer patients receiving palliative care increased from 3.5 to $8.1 \%$ from 2007 to 2011 [23]. However, both studies pointed out the inadequacy of palliative care service for non-cancer patients. In our study, the average length of PCCS was 18.42 days, suggesting 


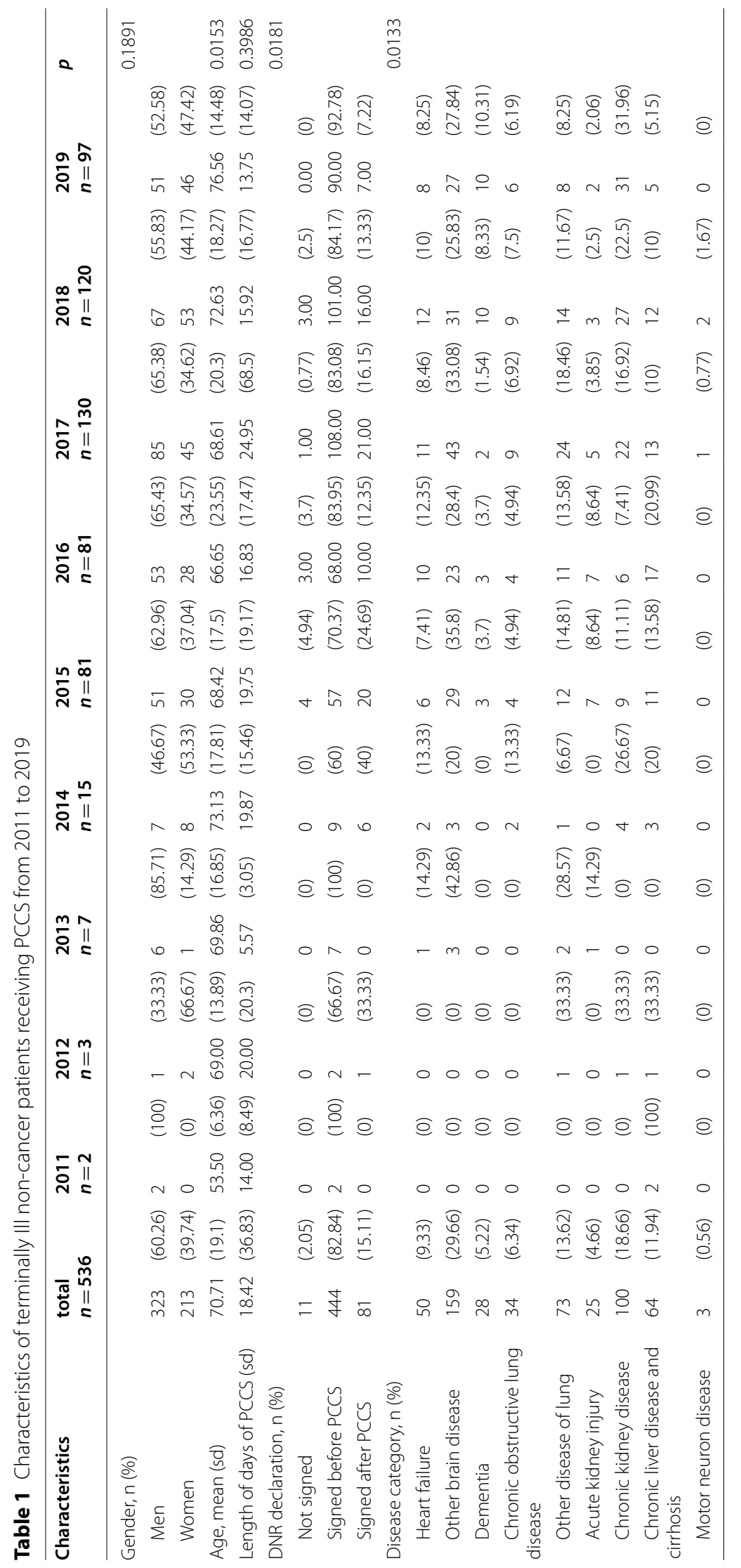




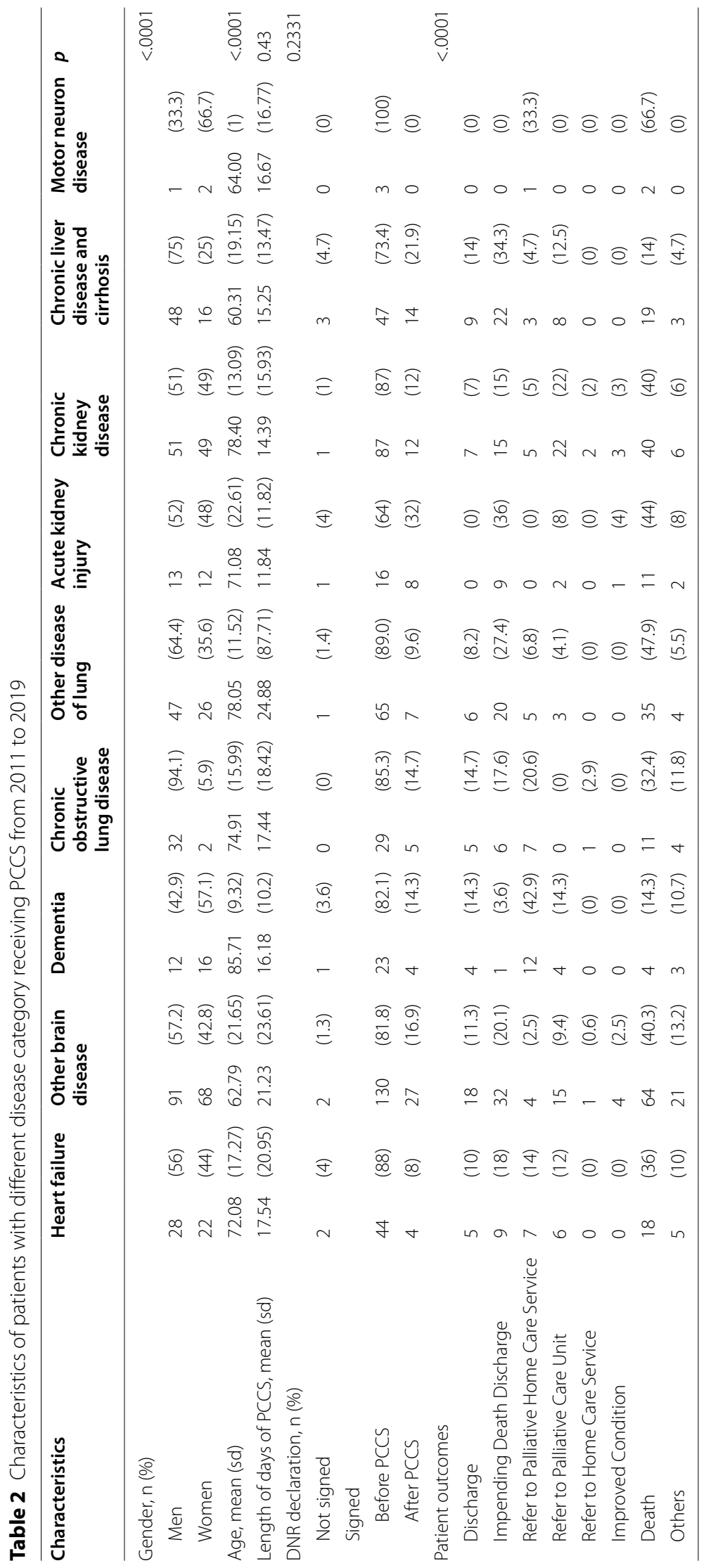




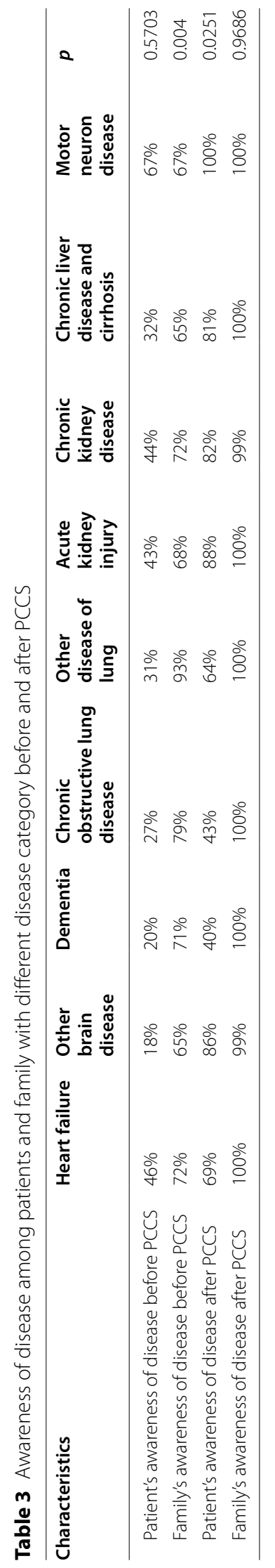




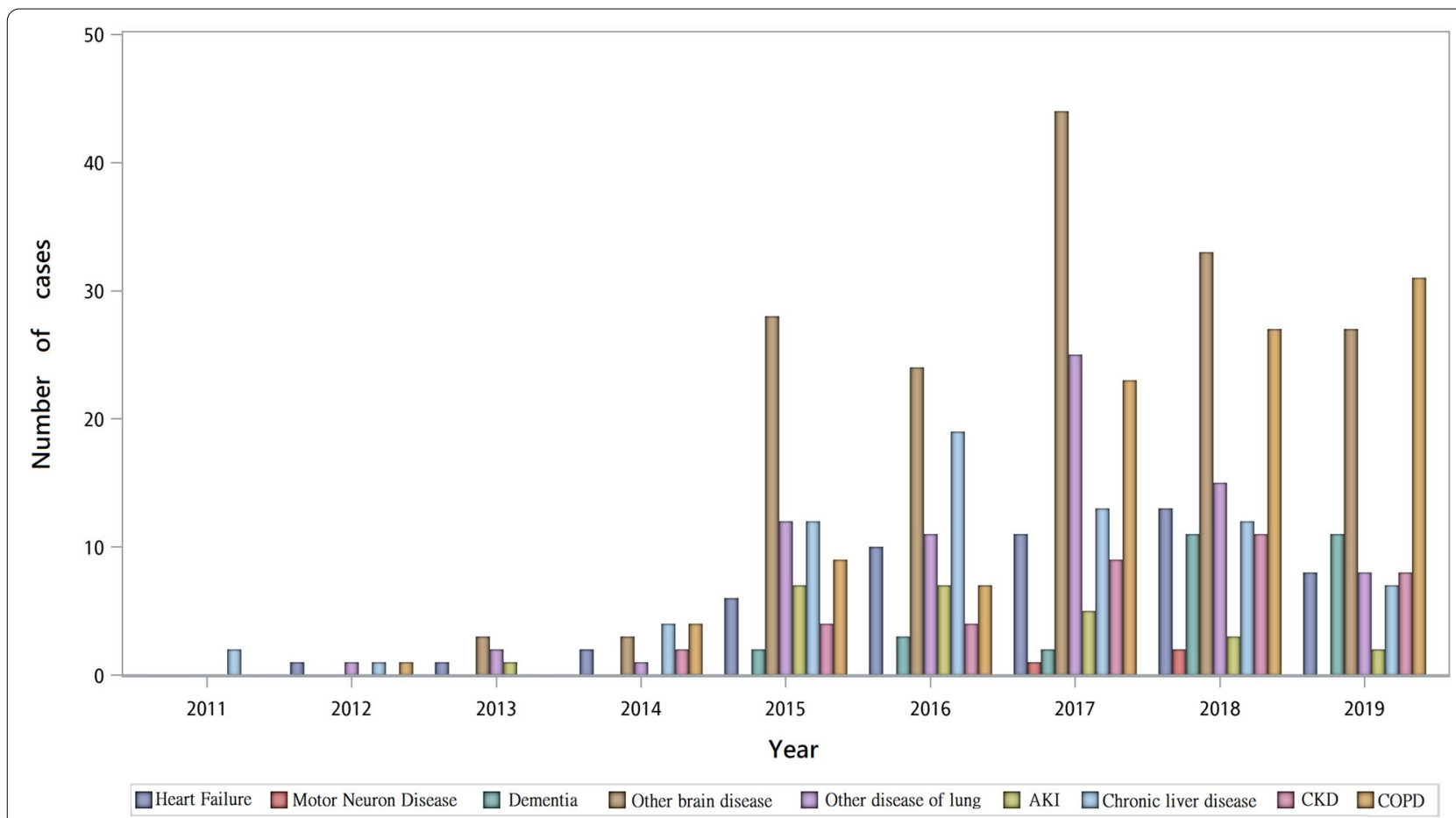

Fig. 1 Trend of terminally ill non-cancer patients receiving PCCS with different disease category from 2011 to 2019

that there is a room for earlier palliative approach. We believe that education and training are key factors for it. In fact, we took an approach of "nurse consultation" for facilitating earlier palliative care in recent years. "Nurse consultation" means that not only physician could initiate the consultation to palliative care team, but also nurses who could do it. Our future study will put emphasis on the effectiveness of this novel approach.

We believe that changes in government policy and the patients' environment led to rising trend in the number of organic brain disease and chronic kidney disease patients receiving PCCS over time. Taiwan is the first East Asian country to legislate withdrawal of life-sustaining treatment in patients at end-of-life. In 2013, the amendment of the Hospice Palliative Care Act allowed the withdrawal of artificial ventilation in terminally ill patients. Since then, the number of patients with an organic brain lesion who received withdrawal of lifesustaining treatment and palliative care increased [24]. In a previous study in Taiwan, Chang et al. discovered that most patients receiving withdrawal of artificial ventilation were non-cancer patients [25]. As for patients with CKD receiving PCCS, a previous study has shown that among patients with advanced CKD receiving palliative care, there were fewer ICU admissions and cardiopulmonary resuscitation sessions compared with those without palliative care [26]. Also, the "Hospice
Palliative Care Act" and "Patient Right to Autonomy Act" helped to establish an environment conductive to better palliative care [27]. In 2016, National Taiwan University Hospital published "Guidelines for hospice palliative care to terminally ill patients with chronic kidney disease at end of life", which suggested integration of early palliative care for all patients with CKD. From our experiences, we believed that PCCS could also have a teaching and training role in the hospital to train hospital healthcare professionals, like physicians and nurses, to provide generalist palliative care to patients before they are terminally ill. Thus, since 2020, we designed a "Life and death experience workshop" for young doctors and nurses to help them understand how to deal with terminally ill patients and families interprofessionally and how PCCS could benefit.

DNR consent increases quality of life for terminally ill patients [28], and a previous study reported that PCCS could significantly increase DNR consent among terminally ill cancer patients [18]. Our results were consistent with a previous study which demonstrated that PCCS increased DNR designation significantly in patients with cancers or non-cancer diseases [29]. A possible explanation could be that cancer patients' and families' awareness of disease and prognosis may have been increased by PCCS [30]. Further study is needed to explore how PCCS improves DNR consideration in non-cancer patients. 


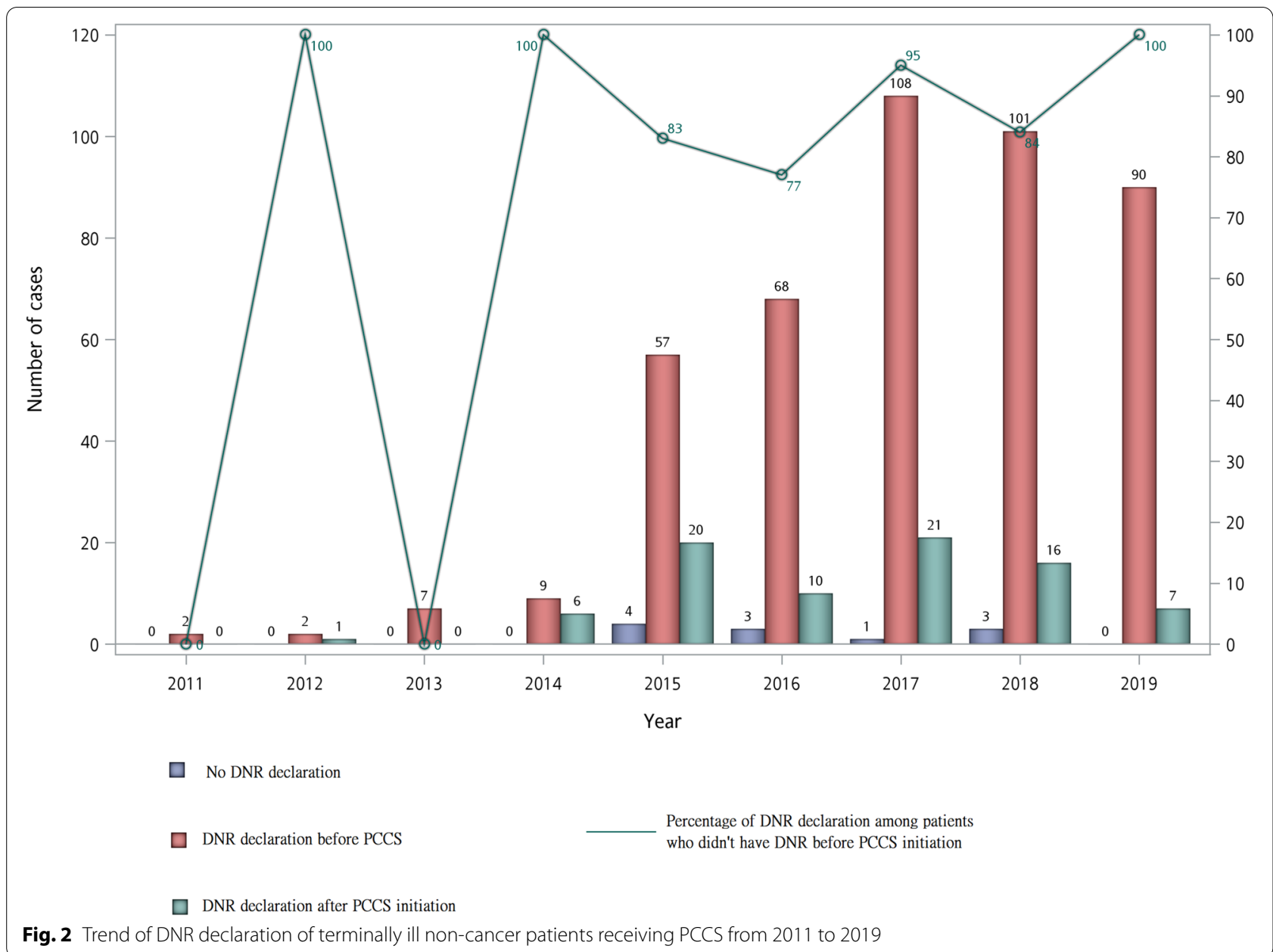

We found that there was a significant difference in awareness of disease between patients and their families, and family members possessed higher awareness of disease compared with the patients. This was an interesting finding because we can logically expect cancer patients in Taiwan to have lower disease awareness [18]. The reason is that doctors traditionally tended to inform family members of a cancer diagnosis rather than the patients themselves $[31,32]$. However, we also found the same situation in non-cancer patients. To the best of our knowledge, there has been no research exploring the relationship between PCCS and disease awareness among non-cancer patients. It has been reported that PCCS can improve family care burden not only in cancer patients but also in non-cancer patients [33]. Future research is warranted to better understand this issue.

After analyzing patient outcomes in non-cancer patients, we found that patients with chronic kidney disease and organic brain disease had more chances to be referred to palliative care unit, while dementia patients were more referred to palliative home care. We believed that this was due to the relatively long trajectory of diseases for patient with chronic kidney disease and organic brain disease, and thus they have more time to think about quality of care with discussion with care providers [34-36]. For demented patients, palliative home care is needed because recurrent biological and psychosocial symptoms [37]. We also found that patients with organic brain disease and chronic liver disease had more opportunity for impending death discharge, and we thought that this could be related to relatively stable condition with less symptom during end-of-life, and family members could accept care at home during end-of-life period.

This study is the first study to analyze the effect of PCCS and the relationships among awareness of disease and patient outcomes in non-cancer patients. However, there were several limitations in this study. First, the data were collected from a single tertiary center in central Taiwan, so there could have been selection bias of participants and the external validity could therefore be limited. Second, data regarding comorbidities and sociodemographic data were lacking, so we could not analyze the 


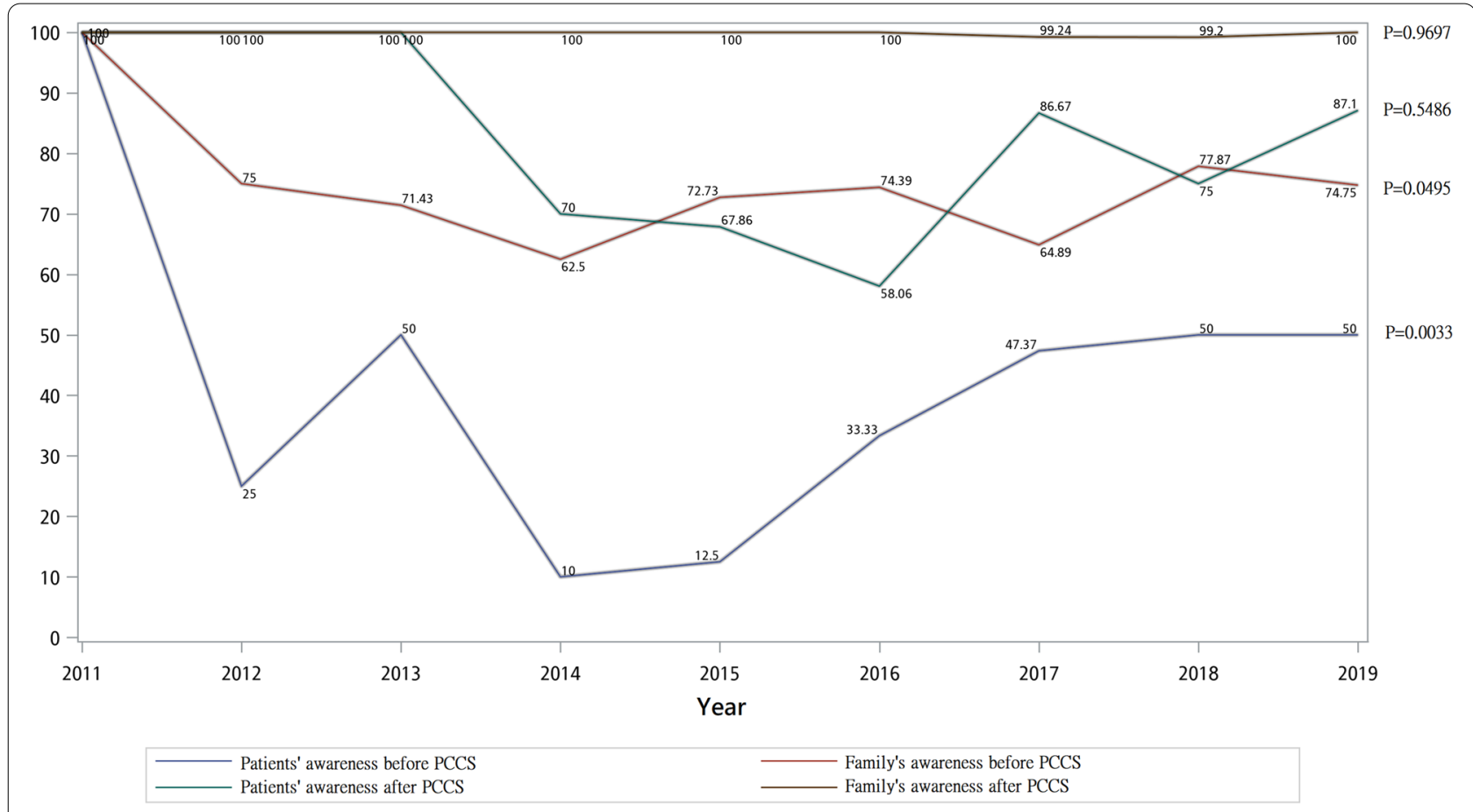

Fig. 3 Trend of non-cancer patients' and family's awareness of disease before and after PCCS from 2011 to 2019

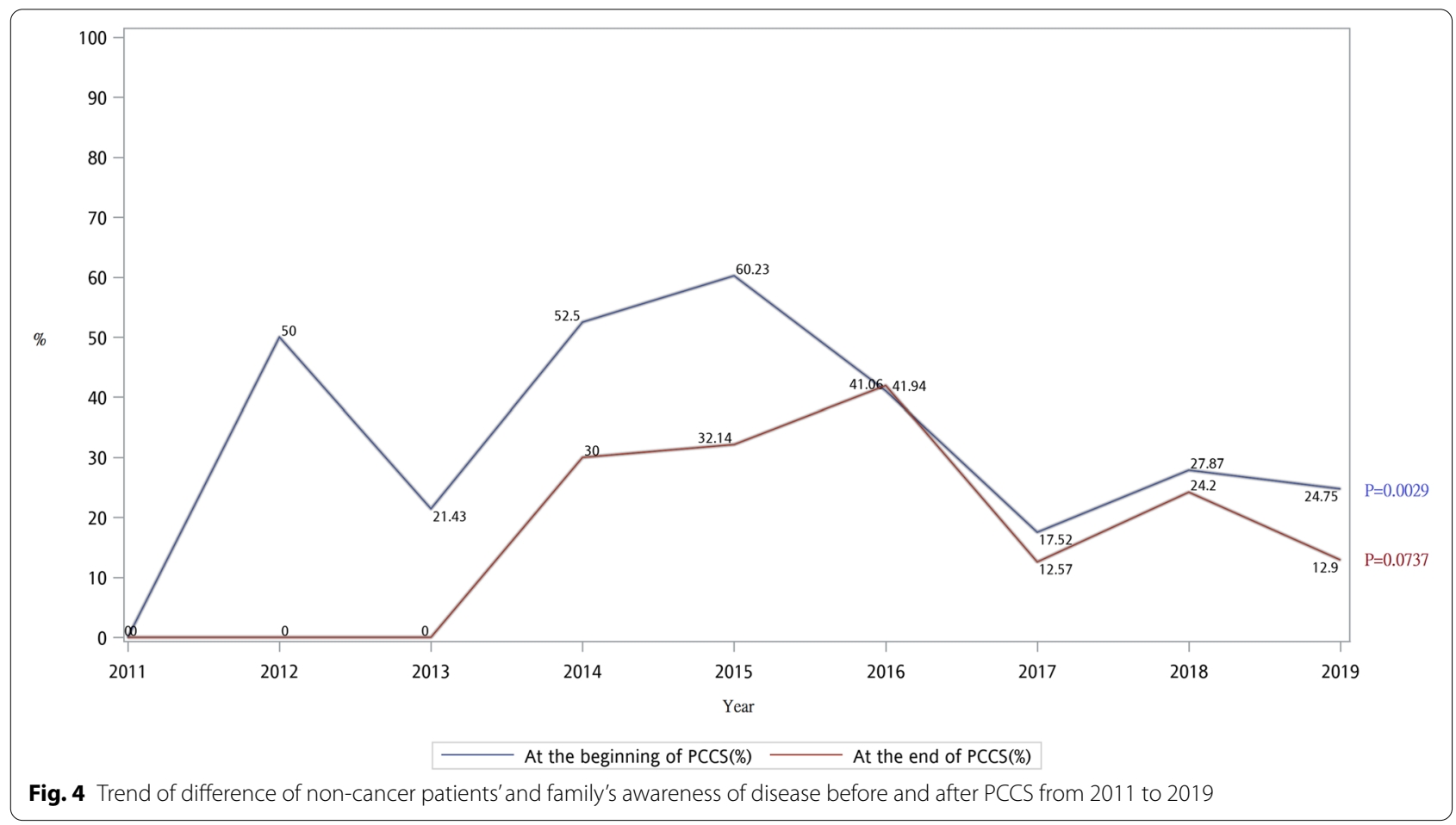

possible effects of comorbidities and sociodemographic condition on awareness of disease, DNR consent, duration of PCCS and patient outcomes.

\section{Conclusion}

This 9-year observational study showed that the trend of PCCS among non-cancer patients changed over the 
duration of the study. The number of terminally ill noncancer patients receiving PCCS during late life rose. PCCS increased awareness of disease in both patients and families, and helped to better prepare terminally ill patients for end-of-life by increasing the likelihood of considering DNR consent. We believe that PCCS will become more important for non-cancer patients as societies in developed nations continue to age. Furthermore, the integration of PCCS into ordinary care for terminally ill noncancer patients is essential for better quality of life.

\author{
Abbreviations \\ DNR: Do-Not-Resuscitate; PCCS: Palliative Care Consultation Service (PCCS); \\ CKD: Chronic Kidney Disease; HPCD: Hospice-Palliative Clinical Database; \\ TCVGH:Taichung Veterans General Hospital; NHI: National Health Insurance; \\ COPD: Chronic Obstructive Lung Disease.
}

\section{Supplementary Information}

The online version contains supplementary material available at https://doi. org/10.1186/s12904-021-00879-z.

Additional file 1: Supplementary Figure 1. Trend of Terminally III NonCancer Patients With/Without PCCS from 2011 to 2019. Supplementary Figure 2. Trend of Terminally III Non-Cancer Patients Receiving PCCS, Palliative Home Care and Palliative Inpatient Care from 2011 to 2019.

\section{Acknowledgements}

With thanks to Clinical Informatics Research \& Development Center of Taichung Veterans General Hospital. We also thank our colleagues from Taichung Veterans General Hospital, Taichung, Taiwan for providing supports in statistical analysis and English editing.

\section{Authors' contributions}

Each author's individual contributions: Conceptualization, W.-M.C.; methodology, W.-M.C.; software, Y.-C.C.; validation, L.-S.L. and L.-H.H.; formal analysis, Y.-C.C. and L.-S.L.; investigation, L.-S.L. and L.-H.H.; resources, C.-L.W. and L.-C.L.; data curation, C.-C.H.; writing —original draft preparation, W.-M.C. and L.-S.L.; writing - review and editing, W.-M.C. and L.-S.L.; supervision, P.-S.H.; project administration, P.-S.H.; funding acquisition, W.-M.C. All authors have read and agreed to the published version of the manuscript.

\section{Funding}

This work was supported by Taichung Veterans General Hospital, Taiwan (Grant number: TCVGH-1107201B awarded to W.-M.C.). The funders had no role in the design of the study; in the collection, analyses, or interpretation of data; in the writing of the manuscript; or in the decision to publish the results.

\section{Availability of data and materials}

The datasets used and analyzed during the current study are not publicity available, but are available from the corresponding author on reasonable request with the permission of Taichung Veterans General Hospital, Taiwan.

\section{Declarations}

Ethics approval and consent to participate

The study was conducted according to the guidelines of the Declaration of Helsinki, and ap-proved by the Institutional Review Board (or Ethics Committee) of Taichung Veterans General Hospital (protocol code TCVGH-IRB CE20362A and date of approval: Dec 2, 2020).

\section{Consent for publication}

The institute review board of Taichung Veterans General Hospital approved this study and deemed that informed consent should be waived owing to the retrospective nature of the study.

\section{Competing interests}

The authors declare no conflicts of interests.

\section{Author details}

'Department of Nursing, Taichung Veterans General Hospital, Taichung, Taiwan. ${ }^{2}$ Technology Transfer and Incubation Center, National Health Research Institutes, Miaoli, Taiwan. ${ }^{3}$ Department of Family Medicine, Taichung Veterans General Hospital, Taichung, Taiwan. ${ }^{4}$ Institute of Medicine, Chung Shan Medical University, Taichung, Taiwan. ${ }^{5}$ Department of Occupational Medicine, Taichung Veterans General Hospital, Taichung, Taiwan. ${ }^{6}$ School of Medicine, National Yang Ming Chiao Tung University, Taipei, Taiwan. ${ }^{7}$ School of Medicine, Chung Shan Medical University, Taichung, Taiwan. ${ }^{8}$ Institue of Health Policy and Management, National Taiwan University, Taipei, Taiwan.

Received: 25 June 2021 Accepted: 8 November 2021

Published online: 25 November 2021

\section{References}

1. Beard JR, Officer A, de Carvalho IA, Sadana R, Pot AM, Michel JP, et al. The World report on ageing and health: a policy framework for healthy ageing. Lancet (London, England). 2016;387(10033):2145-54.

2. Kontis V, Bennett JE, Mathers CD, Li G, Foreman K, Ezzati M. Future life expectancy in 35 industrialised countries: projections with a Bayesian model ensemble. Lancet (London, England). 2017;389(10076):1323-35.

3. Lozano R, Naghavi M, Foreman K, Lim S, Shibuya K, Aboyans V, et al. Global and regional mortality from 235 causes of death for 20 age groups in 1990 and 2010: a systematic analysis for the global burden of disease study 2010. Lancet (London, England). 2012;380(9859):2095-128.

4. Lastrucci V, D'Arienzo S, Collini F, Lorini C, Zuppiroli A, Forni S, et al. Diagnosis-related differences in the quality of end-of-life care: a comparison between cancer and non-cancer patients. PLoS One. 2018;13(9):e0204458.

5. Alexander K, Goldberg J, Korc-Grodzicki B. Palliative care and symptom Management in Older Patients with Cancer. Clin Geriatr Med. 2016;32(1):45-62.

6. Greer JA, Applebaum AJ, Jacobsen JC, Temel JS, Jackson VA. Understanding and addressing the role of coping in palliative Care for Patients with Advanced Cancer. J Clin Oncol. 2020;38(9):915-25.

7. Haun MW, Estel S, Rücker G, Friederich HC, Villalobos M, Thomas M, et al. Early palliative care for adults with advanced cancer. Cochrane Database Syst Rev. 2017:6(6):Cd011129.

8. Ullrich A, Ascherfeld L, Marx G, Bokemeyer C, Bergelt C, Oechsle K. Quality of life, psychological burden, needs, and satisfaction during specialized inpatient palliative care in family caregivers of advanced cancer patients. BMC Palliative Care. 2017;16(1):31.

9. Gadoud A, Kane E, Oliver SE, Johnson MJ, Macleod U, Allgar V. Palliative care for non-cancer conditions in primary care: a time trend analysis in the UK (2009-2014). BMJ Support Palliat Care. 2020;bmjspcare-2019-001833.

10. Luddington L, Cox S, Higginson I, Livesley B. The need for palliative care for patients with non-cancer diseases: a review of the evidence. Int J Palliat Nurs. 2001;7(5):221-6.

11. Chochinov HM, Johnston W, McClement SE, Hack TF, Dufault B, Enns M, et al. Dignity and distress towards the end of life across four non-Cancer populations. PLoS One. 2016;11(1):e0147607.

12. Quinn KL, Stukel T, Stall NM, Huang A, Isenberg S, Tanuseputro P, et al. Association between palliative care and healthcare outcomes among adults with terminal non-cancer illness: population based matched cohort study. BMJ (Clinical research ed). 2020;370:m2257.

13. Higginson IJ, Bausewein C, Reilly CC, Gao W, Gysels M, Dzingina M, et al. An integrated palliative and respiratory care service for patients with advanced disease and refractory breathlessness: a randomised controlled trial. Lancet Respir Med. 2014;2(12):979-87. 
14. Hung YS, Chen CH, Yeh KY, Chang H, Huang YC, Chang CL, et al. Potential benefits of palliative care for polysymptomatic patients with late-stage nonmalignant disease in Taiwan. Journal of the Formosan medical association =. Taiwan yi zhi. 2013;112(7):406-15.

15. Elsayem A, Calderon BB, Camarines EM, Lopez G, Bruera E, Fadul NA. A month in an acute palliative care unit: clinical interventions and financial outcomes. Am J Hosp Palliat Care. 2011;28(8):550-5.

16. Reymond L, Parker G, Gilles L, Cooper K. Home-based palliative care. Aust J Gen Pract. 2018;47(11):747-52.

17. Engel PT, Thavayogarajah T, Görlich D, Lenz P. Establishment of a Palliative Care Consultation Service (PCCS) in an Acute Hospital Setting. Int J Environ Res Public Health. 2020;17(14):4977.

18. Lu CY, Shen WC, Kao CY, Wang HM, Tang SC, Chin TL, et al. Impact of palliative care consultation service on terminally ill Cancer patients: a 9-year observational cohort study in Taiwan. Medicine. 2016;95(10):e2981.

19. Basedow-Rajwich B, Montag T, Duckert A, Schulz C, Rajwich G, Kleiter I, et al. Mobile palliative care consultation service (PCCS): overview of hospice and palliative care evaluation (HOPE) data on in-patients with end-stage Cancer, multiple sclerosis, and noncancer, nonneurological disease from 4 PCCS centers in Germany in 2013. Palliat Care. 2018;11:1178224218785139.

20. Shih TC, Chang HT, Lin MH, Chen CK, Chen TJ, Hwang SJ. Differences in do-not-resuscitate orders, hospice care utilization, and late referral to hospice care between cancer and non-cancer decedents in a tertiary Hospital in Taiwan between 2010 and 2015: a hospital-based observational study. BMC Palliat Care. 2018;17(1):18.

21. Chen RC. Hospice palliative care policy in Taiwan: past developments and future perspectives. Hu Li Za Zhi. 2015;62(2):13-7.

22. Yang PC, Shih MJ, Liu YA, Hsu YC, Chang HT, Lin MH, et al. Web Search Trends of Implementing the Patient Autonomy Act in Taiwan. Healthcare (Basel, Switzerland). 2020;8(3):353.

23. Hess S, Stiel S, Hofmann S, Klein C, Lindena G, Ostgathe C. Trends in specialized palliative care for non-cancer patients in Germany--data from the national hospice and palliative care evaluation (HOPE). Eur J Intern Med. 2014;25(2):187-92.

24. Hung YS, Lee SH, Hung CY, Wang CH, Kao CY, Wang HM, et al. Clinical characteristics and survival outcomes of terminally ill patients undergoing withdrawal of mechanical ventilation. J Formos Med Assoc. 2018;117(9):798-805.

25. Chang HT, Lin MH, Chen CK, Chen TJ, Hwang SJ. Aggressive end-of-life care and symptom relief treatments in terminally ill patients who had discussed withdrawal of mechanical ventilation: a hospital-based observational study. Am J Hosp Palliat Care. 2020;37(11):897-903.

26. Lai CF, Hsu SH, Huang SJ. Incorporating palliative care into the Dialysis unit affects patterns near the end of life. Mayo Clin Proc. 2015;90(9):1307-9.
27. Wu MY, Wu MS. Taiwan renal care system: A learning health-care system. Nephrology (Carlton, Vic). 2018;23(Suppl 4):112-5.

28. Wang CL, Lin CY, Huang CC, Lin CS, Hu CC, Hwang SF, et al. Do-notresuscitate status is correlated with the prescribed use of systemic strong opioid analgesics in patients with terminal cancer: an observational study. Support Care Cancer. 2019;27(12):4507-13.

29. Hung YS, Chang H, Wu WS, Chen JS, Chou WC. A comparison of cancer and noncancer patients who receive palliative care consultation services. Am J Hosp Palliat Care. 2013;30(6):558-65.

30. Kao CY, Wang HM, Tang SC, Huang KG, Jaing TH, Liu CY, et al. Predictive factors for do-not-resuscitate designation among terminally ill cancer patients receiving care from a palliative care consultation service. J Pain Symptom Manag. 2014;47(2):271-82.

31. Tang ST, Liu TW, Lai MS, Liu LN, Chen CH. Concordance of preferences for end-of-life care between terminally ill cancer patients and their family caregivers in Taiwan. J Pain Symptom Manag. 2005;30(6):510-8.

32. Li JY, Liu C, Zou LQ, Huang MJ, Yu CH, You GY, et al. To tell or not to tell: attitudes of Chinese oncology nurses towards truth telling of cancer diagnosis. J Clin Nurs. 2008;17(18):2463-70.

33. Wu LF, Lin C, Hung YC, Chang LF, Ho CL, Pan HH. Effectiveness of palliative care consultation service on caregiver burden over time between terminally ill cancer and non-cancer family caregivers. Support Care Cancer. 2020;28(12):6045-55.

34. Wasylynuk BA, Davison SN. Palliative care in patients with advanced chronic kidney disease. CANNT J. 2015;25(3):28-32.

35. Holley JL. Palliative care in end-stage renal disease: illness trajectories, communication, and hospice use. Adv Chronic Kidney Dis. 2007;14(4):402-8.

36. Mead GE, Cowey E, Murray SA. Life after stroke - is palliative care relevant? A better understanding of illness trajectories after stroke may help clinicians identify patients for a palliative approach to care. Int J Stroke. 2013;8(6):447-8.

37. Miranda R, Smets T, De Schreye R, Faes K, Van Den Noortgate N, Cohen J, et al. Improved quality of care and reduced healthcare costs at the endof-life among older people with dementia who received palliative home care: a nationwide propensity score-matched decedent cohort study. Palliat Med. 2021;2692163211019321:1701-12

\section{Publisher's Note}

Springer Nature remains neutral with regard to jurisdictional claims in published maps and institutional affiliations.
Ready to submit your research? Choose BMC and benefit from:

- fast, convenient online submission

- thorough peer review by experienced researchers in your field

- rapid publication on acceptance

- support for research data, including large and complex data types

- gold Open Access which fosters wider collaboration and increased citations

- maximum visibility for your research: over $100 \mathrm{M}$ website views per year

At BMC, research is always in progress.

Learn more biomedcentral.com/submissions 